\title{
Reclassification of Mongolian Diffuse Gliomas According to the Revised 2016 World Health Organization Central Nervous System Tumor Classification
}

\author{
Enkhee Ochirjav, Bayarmaa Enkhbat, Tuul Baldandorj, Gheeyoung Choe ${ }^{1}$ \\ Department of Pathology, School of Biomedicine, Mongolian National University of Medical Sciences, Ulaanbaatar, Mongolia; \\ ${ }^{1}$ Department of Pathology, Seoul National University Bundang Hospital, Seoul National University College of Medicine, Seongnam, Korea
}

\begin{abstract}
Background: The 2016 World Health Organization (WHO) classification of central nervous system (CNS) tumors has been modified to incorporate the IDH mutation and 1p/19q co-deletion in the diagnosis of diffuse gliomas. In this study, we aimed to evaluate the feasibility and prognostic significance of the revised 2016 WHO classification of CNS tumors in Mongolian patients with diffuse gliomas. Methods: A total of 124 cases of diffuse gliomas were collected, and tissue microarray blocks were made. IDH1 mutation was tested using immunohistochemistry, and 1p/19q co-deletion status was examined using fluorescence in situ hybridization analysis. Results: According to the 2016 WHO classification, 124 cases of diffuse brain glioma were reclassified as follows: 10 oligodendroglioma, IDH ${ }^{\text {mut }}$ and

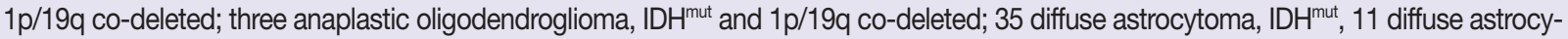
toma, IDH ${ }^{\text {wt }}$, not otherwise specified (NOS); 22 anaplastic astrocytoma, IDH ${ }^{\text {mut }}$, eight anaplastic astrocytoma, IDH ${ }^{\text {wt }}$, NOS; and 35 glioblastoma, IDH't, NOS, respectively. The 2016 WHO classification presented better prognostic value for overall survival in patients with grade II tumors than traditional histological classification. Among patients with grade II tumors, those with oligodendroglioma IDH ${ }^{\text {mut }}$ and $1 p / 19 q$ co-deleted and diffuse astrocytoma IDH ${ }^{\text {mut }}$ showed significantly higher survival than those with diffuse astrocytoma IDH ${ }^{\text {wt }}$, NOS $(p<.01)$. Conclusions: Mongolian diffuse gliomas could be reclassified according to the new 2016 WHO classification. Reclassification revealed substantial changes in diagnosis of both oligodendroglial and astrocytic entities. We have confirmed that the revised 2016 WHO CNS tumor classification has prognostic significance in Mongolian patients with diffuse gliomas, especially those with grade II tumors.
\end{abstract}

Key Words: World Health Organization; Classification; Glioma; Isocitrate dehydrogenase; Chromosome deletion

Received: May 3, 2019 Revised: July 12, 2019 Accepted: July 15, 2019

Corresponding Author: Gheeyoung Choe, MD, Department of Pathology, Seoul National University Bundang Hospital, Seoul National University College of Medicine, 82 Gumi-ro 173beon-gil, Bundang-gu, Seongnam 13620, Korea

Tel: +82-31-787-7711, Fax: +82-31-787-4012, E-mail: gychoe@snubh.org

Gliomas are the primary tumors that develop in the central nervous system (CNS). Diffuse glioma is defined by indefinite border of tumor mass and is considered as a more aggressive form of gliomas. ${ }^{1,2}$ Diagnosis of glioma was mainly based on the 2007 World Health Organization (WHO) classification, which considered increased cellularity, nuclear atypia, mitotic activity, microvascular proliferation, and necrosis for malignant criteria (histologic grade of gliomas). ${ }^{3}$ Histological evaluation for grading is important for the treatment of diffuse gliomas; however, histologic grade is not the only prognostic determinant in diffuse gliomas. The chromosomal aberrations such as deletion and mutation are common in gliomas, ${ }^{4}$ and oligodendroglial phenotype gliomas with 1p/19q co-deletion tend to have a better prognosis and respond well to chemotherapy and concurrent chemoradiation therapy. ${ }^{5}$ Moreover, gliomas with IDH mutation have more favorable prognosis than IDH-wildtype gliomas. ${ }^{2}$ Therefore, at the Haarlem meeting in 2014, multidisciplinary specialists concluded that molecular information should be incorporated into the diagnosis of gliomas to make more integrated diagnosis. ${ }^{6}$

The updated 2016 WHO classification of CNS tumors incorporated molecular features such as IDH mutation and chromosome $1 \mathrm{p} / 19 \mathrm{q}$ co-deletion into the diagnosis of gliomas. ${ }^{7}$ Previous classification published in 2007 was mainly based on the histological and immunohistochemical features of the tumor. ${ }^{3,8}$ The new 2016 WHO CNS tumor classification led to substantial 
changes in the diagnosis of diffuse gliomas depending on the presence or absence of IDH mutation and 1p/19q co-deletion. ${ }^{5,9}$ Diffuse glioma with both IDH mutation and 1p/19q co-deletion is referred to as oligodendroglioma, IDH-mutant, and $1 \mathrm{p} / 19 \mathrm{q}$ co-deleted, which has a better prognosis compared with intact cases. ${ }^{10}$ Although a glioma shows oligodendroglioma-like histologic feature, it is no longer classified as an oligodendroglial tumor if neither IDH mutation nor 1p/19q co-deletion is present. Diffuse astrocytic gliomas are classified as an IDH-mutant or IDHwildtype according to the IDH mutation status. IDH-wildtype gliomas with epidermal growth factor receptor (EGFR) amplification are considered to have more aggressive behavior like glioblastoma. ${ }^{11}$ Diffuse astrocytoma or oligodendroglioma, which has been diagnosed based on histologic features only without molecular testing, is classified into the "NOS (not otherwise specified)" category. ${ }^{11,12}$ cIMPACT-NOW (the Consortium to Inform Molecular and Practical Approaches to CNS Tumor Taxonomy) was established to provide a forum to evaluate and recommend proposed changes to future CNS tumor classifications. Recently, CIMPACT clarified the use of the term NOS and proposed the use of an additional term "NEC (not elsewhere classified)" as well. ${ }^{13}$ For an NOS designation, diagnostic information (histological or molecular) necessary to assign more specific WHO diagnosis is not available. For an NEC designation, necessary diagnostic testing has been successfully performed, but the results do not readily allow for a WHO 2016 diagnosis. In some instances, this will be caused by a mismatch between clinical, histological, immunohistological and/or genetic features; in others, the results may support a new or emerging entity that is not yet included in the WHO classification. ${ }^{13}$

In this study, we aimed to reclassify diffuse brain gliomas according to the revised 2016 WHO classification of CNS tumors in Mongolian patients with brain gliomas and to evaluate the prognostic significance of the revised 2016 WHO classification of CNS tumors.

\section{MATERIALS AND METHODS}

\section{Tumor samples}

Data of 124 patients who have been diagnosed with diffuse gliomas according to the WHO 2007 criteria in the National Center for Pathology of Mongolia between January 2006 and December 2017 were obtained in this study. We marked the representative tumor areas on hematoxylin and eosin (H\&E)stained sections. Tumor areas containing viable tumor cell infiltration over $60 \%$ without necrosis or hemorrhage were selected.
Corresponding areas were identified on the formalin-fixed, paraffin-embedded archival blocks, and we constructed tissue microarray (TMA) blocks using 3-mm cores. Each TMA block was verified by H\&E staining to determine whether each core has intact glioma tissue.

\section{IDH mutation status}

In immunohistochemistry, 4 - $\mu \mathrm{m}$-thick tissue sections were deparaffinized in xylene and hydrated by immersing them in a series of graded ethanol. Antigen retrieval was performed in the microwave by placing the sections in epitope retrieval solution (0.01 M citrate buffer, $\mathrm{pH}$ 6.0) for 20 minutes; endogenous peroxidase was inhibited by immersing the sections in $0.3 \%$ hydrogen peroxide for 10 minutes. Sections were then incubated with IDH1 (1:100, Dianova, Hamburg, Germany) antibody. Then, an OptiView DAB IHC Detection Kit (Ventana Medical Systems, Tucson, AZ, USA) was used following the manufacturer's recommendations in conjunction with an automated staining procedure using Benchmark XT (Ventana Medical Systems). Finally, the samples were counterstained with hematoxylin, dehydrated, mounted, and evaluated under a light microscope equipped with an Olympus CX21 camera (Tokyo, Japan) (Fig. 1).

\section{Fluorescence in situ hybridization}

Fluorescence in situ hybridization (FISH) with Vysis probes was used to assess 1p/19q status. TMA sections were deparaffinized with xylene, incubated with $0.3 \%$ pepsin in $10 \mathrm{mM} \mathrm{HCl}$ at $37^{\circ} \mathrm{C}$ for 10 minutes, and then denatured at $85^{\circ} \mathrm{C}$ for 10 minutes. FISH analyses were performed on deparaffinized sections with a dual-color approach for chromosomes 1 and 19, respectively. Target probes were hybridized to subtelomeric $1 \mathrm{p} 36$ and $19 \mathrm{q} 13$ in combination with control probes on 1q25 and 19 p13, respectively. For evaluation, the signal ratio in 50-100 adjacent, non-overlapping interphase nuclei was assessed, and the results were expressed as percentage (Fig. 2).

\section{Statistical analysis}

Continuous data were presented as the mean \pm standard deviation, while categorical data were presented as frequencies and percentages. Continuous variables that were not normally distributed (as evaluated by Kolmogorov-Smirnov tests) were presented as medians and 25 th and 75 th percentiles. Differences in baseline characteristics were estimated using the chi-square test.

Overall survival (OS) was defined as the time from the date of surgery to death from any cause. The discriminative value of the 2007 and 2016 WHO classifications were estimated using Cox 

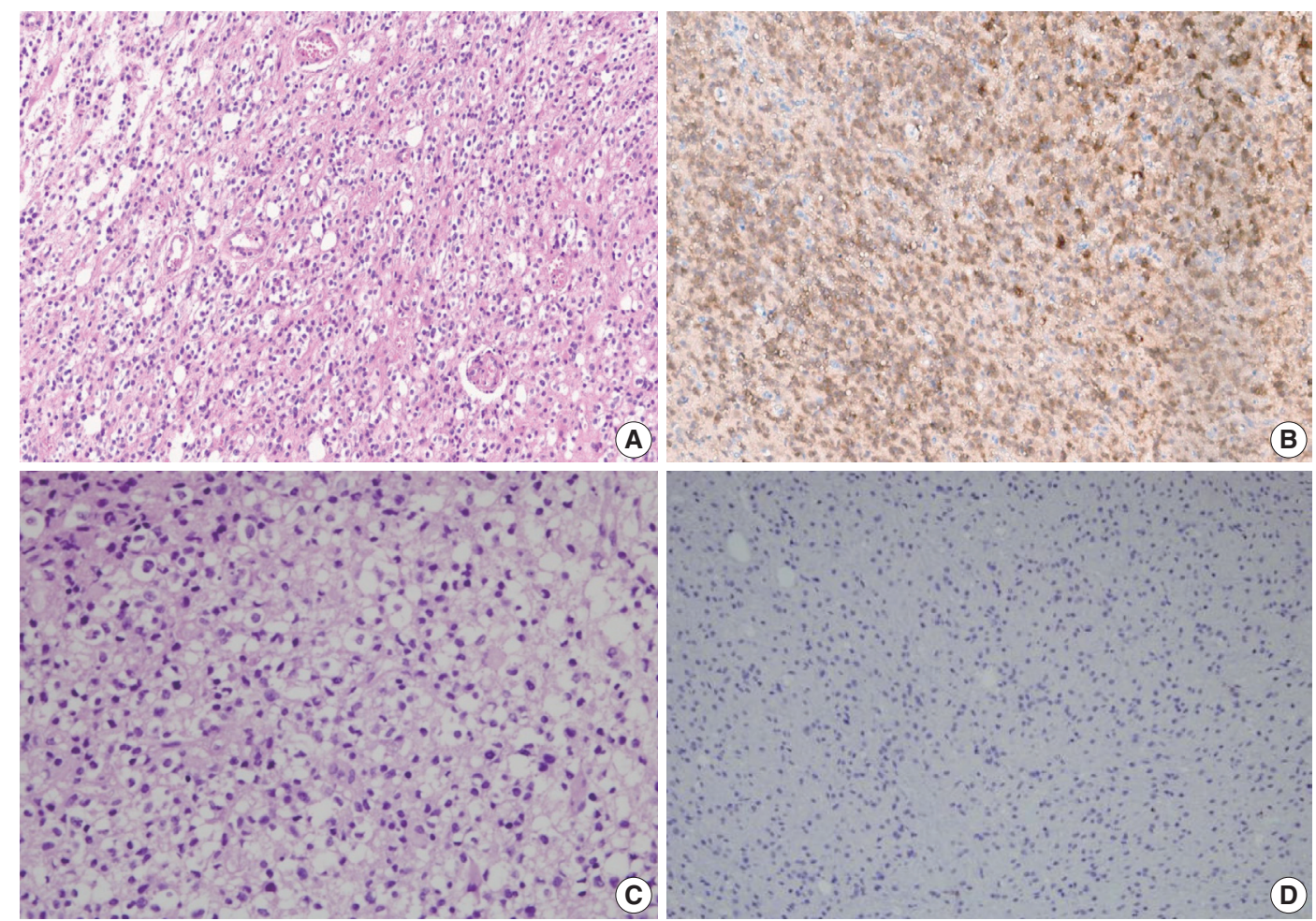

Fig. 1. IDH1 immunohistochemical staining of diffuse brain glioma. (A) Definite fried egg appearance (perinuclear halo) of oligodendroglioma in hematoxylin and eosin (H\&E) staining $\times 100$. (B) The tumor cells express IDH1 in the cytoplasm by immunohistochemistry. The unstained cells in the brain parenchyma represent non-neoplastic cells. (C) H\&E staining of anaplastic astrocytoma with pleomorphic nucleus $\times 100$. (D) The tumor cells are negative for IDH1 immunostaining.
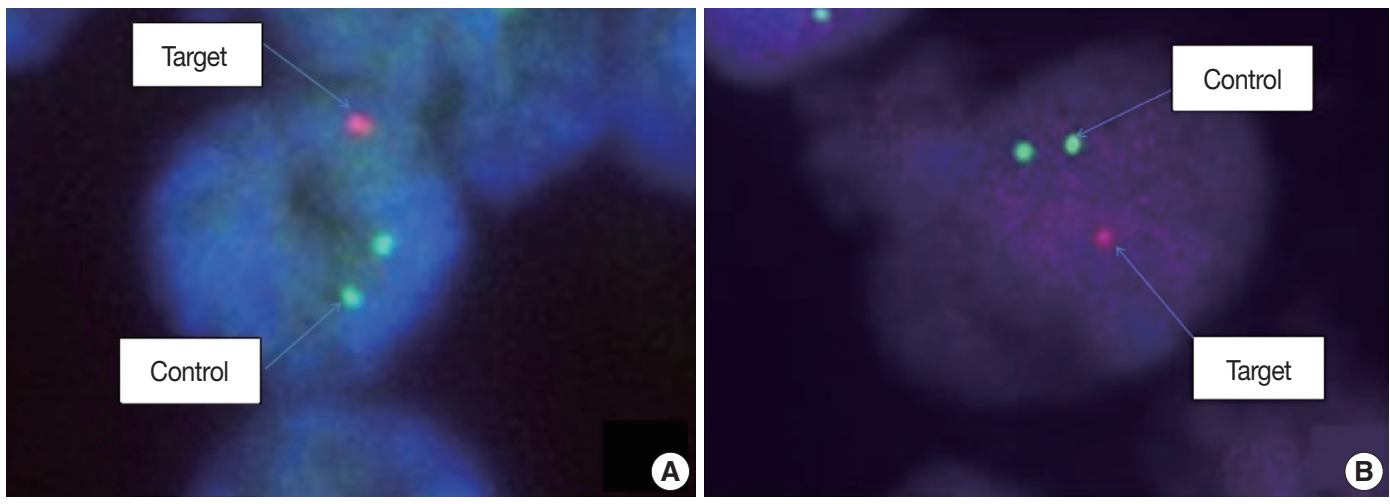

Fig. 2. Fluorescence in situ hybridization (FISH) analysis of $1 \mathrm{p} / 19 \mathrm{q}$ co-deletion on formalin-fixed paraffin-embedded specimen: (A) FISH preparation showing $1 p$ deletion in an oligodendroglioma. A tumor cell in 1p deletion status is clearly seen, with a 2:1 ratio of control (green) signals and target (red) signal. (B) FISH preparation showing 19q deletion in the same oligodendroglioma case. One cell is labeled showing one red signal for the 19q test probe and two green signals for the 19q control probe, indicating loss of one copy of 19q.

proportional hazard regression model for all-cause mortality. The Kaplan-Meier method was used to estimate survival distributions.

All statistical tests were two-sided, and a p-value of $<.05$ was considered significant. All statistical analyses were conducted using SPSS ver. 22.0 (IBM Corp., Armonk, NY, USA).

\section{Ethics statement}

All procedures performed in the current study were approved by the Institutional Review Board of Seoul National University Bundang Hospital (B-1703/385-302) and Research Ethics Committee of the Mongolian National University of Medical Sciences (MNUMS) (2017/3-201702) in accordance with the 1964 Helsinki declaration and its later amendments. Formal 
written informed consent was not required with a waiver by the Institutional Review Board of Seoul National University Bundang Hospital and Research Ethics Committee of the MNUMS.

\section{RESULTS}

\section{Patient characteristics}

Data of 124 patients diagnosed with diffuse brain glioma between January 2006 and December 2017 were collected (men, $48.4 \%$ and women, 51.6\%). The median age at diagnosis was 41 years (interquartile range [IQR], 29 to 52), and the median follow-up period was 8 months (IQR, 4 to 15 ). Grade II, III, and IV tumors developed in 45.2\% ( $\mathrm{n}=56), 26.6 \%(\mathrm{n}=33)$, and $28.2 \%(\mathrm{n}=35)$ of patients, respectively. Approximately $46.8 \%(\mathrm{n}=58)$ of patients underwent complete tumor resection, while $53.2 \%$ ( $n=66$ ) underwent partial tumor resection. According to the 2007 WHO classification, $23.4 \%(n=29)$ of patients developed diffuse astrocytoma; $21 \%(\mathrm{n}=26)$, oligodendroglioma; $0.8 \%(\mathrm{n}=1)$, oligoastrocytoma; $13.7 \%(\mathrm{n}=17)$, anaplastic astro-

Table 1. Patient characteristics

\begin{tabular}{lc}
\hline Variable & No. (\%) \\
\hline Age, median (IQR, yr) & $41(29-52)$ \\
Sex & \\
Male & $60(48.4)$ \\
Female & $64(51.6)$ \\
WHO tumor grade & \\
Grade II & $56(45.2)$ \\
Grade III & $33(26.6)$ \\
Grade IV & $35(28.2)$ \\
Type of surgery & \\
Complete resection & $58(46.8)$ \\
Partial resection & $66(53.2)$ \\
Immunohistochemical and molecular changes & \\
p53 expression> 10\% & $53(42.7)$ \\
PTEN loss & $73(54.9)$ \\
EGFR amplification & $43(32.8)$ \\
WT1 high expression & $39(29.3)$ \\
IDH1 mut & $70(56.5)$ \\
1p/19q co-deletion & $13(10.5)$ \\
Histological diagnosis according to the 2007 WHO classification \\
Astrocytic tumors \\
Diffuse astrocytoma (grade II) \\
Anaplastic astrocytoma (grade III) \\
Glioblastoma (grade IV) \\
Oligodendroglial tumors & $29(23.4)$ \\
$\quad$ Oligodendroglioma (grade II) & $17(13.7)$ \\
$\quad$ Anaplastic oligodendrogloima (grade III) & $35(28.2)$ \\
Mixed oligoastrocytoma (grade II) & \\
\hline
\end{tabular}

IQR, interquartile range; WHO, World Health Organization; PTEN, phosphatase and tensin homolog; EGFR, endothelial growth factor receptor; WT1, Wilms tumor 1; IDH1 ${ }^{\text {mut }}$, isocitrate dehydrogenase 1-mutant. cytoma; $12.9 \%$ ( $\mathrm{n}=16$ ), anaplastic oligodendroglioma; and $28.2 \%$ $(\mathrm{n}=35)$, glioblastoma. Patients' baseline characteristics are summarized in Table 1.

\section{Molecular data and tumor reclassification according to the 2016 WHO classification}

A total of 124 patients underwent FISH test; however, 32 patients, whose tissue samples were archived before 2012, showed no signal on FISH test. Therefore, 92 patients were analyzed for 1p/19q co-deletion by FISH. About 32 patients, with absence of signal on FISH test, were reclassified based on histological pattern and IDH1 mutation status only without $1 \mathrm{p} / 19 \mathrm{q}$ co-deletion information. Immunohistochemical staining of IDH1 was performed on all 124 patients. 1p/19q co-deletion was detected in 13 of 92 patients (10.5\%) who underwent FISH test, and IDH1 mutation was detected in 70 of 124 IDH1 immunostained patients $(56.5 \%)$.

The molecular studies performed for reclassification have limitations in this study. The updated 2016 WHO classification of CNS tumors recommends full assessment of IDH mutation status (sequence analysis for $I D H 1$ codon 132 and IDH2 codon 172) in cases of diffuse gliomas that are immunohistochemically negative for IDH1 R132H mutation. In the present study, IDH mutation status has been investigated only by immunohistochemistry, and no further molecular analysis for IDH mutation was performed. Therefore, we reclassified IDH1 immuno-negative diffuse gliomas as diffuse gliomas, IDH-wildtype, NOS. Among 32 cases which showed technical failure for FISH test, neither immunohistochemical expression of IDH1 nor morphological phenotype of oligodendroglioma was detected. Therefore, there was no case of oligodendroglioma/anaplastic oligodendroglioma, IDH-mutant, NOS.

According to the updated 2016 WHO classification, diffuse astrocytomas $(\mathrm{n}=29)$ were reclassified into 18 diffuse astrocytomas IDH ${ }^{\text {mut }}$ (IDH-mutant), nine diffuse astrocytomas IDH ${ }^{\text {wt }}$ (IDH-wildtype), NOS, and two oligodendrogliomas IDH ${ }^{\text {mut }}$ and $1 \mathrm{p} / 19 \mathrm{q}$ co-deleted. Anaplastic astrocytomas $(\mathrm{n}=17)$ were reclassified into eight anaplastic astrocytomas $\mathrm{IDH}^{\text {mut }}$, eight anaplastic astrocytomas IDH ${ }^{\mathrm{wt}}$, NOS, and one anaplastic oligodendroglioma IDH ${ }^{\text {mut }}$ and 1p/19q co-deleted. Glioblastomas (n =35) were all reclassified into glioblastoma $\mathrm{IDH}^{\mathrm{wt}}$, NOS. Oligodendrogliomas $(\mathrm{n}=26)$ were reclassified into 16 diffuse astrocytomas IDH ${ }^{\text {mut }}$, eight oligodendrogliomas IDH ${ }^{\text {mut }}$ and $1 \mathrm{p} / 19 \mathrm{q}$ co-deleted, and two diffuse astrocytomas IDH ${ }^{\mathrm{wt}}$, NOS. Anaplastic oligodendrogliomas $(\mathrm{n}=16)$ were reclassified into 14 anaplastic astrocytomas IDH ${ }^{\text {mut }}$ and two anaplastic oligodendrogli- 
Table 2. Summary of subgroups of diffuse gliomas according to updated 2016 WHO classification

\begin{tabular}{|c|c|c|}
\hline Histological diagnosis (WHO 2007) & Integrated diagnoses (WHO 2016) & No. \\
\hline \multirow[t]{3}{*}{ Diffuse astrocytoma $(n=29)$} & Diffuse astrocytoma IDH'mut & 18 \\
\hline & Diffuse astrocytoma IDH ${ }^{\mathrm{wt}}$, NOS & 9 \\
\hline & Oligodendroglioma IDH ${ }^{\text {mut }}, 1 p / 19 q$ co-deleted & 2 \\
\hline \multirow[t]{3}{*}{ Anaplastic astrocytoma $(n=17)$} & Anaplastic astrocytoma IDH mut & 8 \\
\hline & Anaplastic astrocytoma IDH'wt, NOS & 8 \\
\hline & Anaplastic oligodendroglioma IDHmut, $1 p / 19 q$ co-deleted & 1 \\
\hline Glioblastoma $(n=35)$ & Glioblastoma IDH'wt, NOS & 35 \\
\hline \multirow[t]{3}{*}{ Oligodendroglioma $(n=26)$} & Diffuse astrocytoma IDH $\mathrm{Imut}^{\mathrm{m}}$ & 16 \\
\hline & Oligodendroglioma IDH'mut, $1 p / 19 q$ co-deleted & 8 \\
\hline & Diffuse astrocytoma IDH'wt, NOS & 2 \\
\hline \multirow{2}{*}{ Anaplastic oligodendroglioma $(n=16)$} & Anaplastic astrocytoma IDH ${ }^{\text {mut }}$ & 14 \\
\hline & Anaplastic oligodendroglioma IDHmut, $1 p / 19 q$ co-deleted & 2 \\
\hline Mixed oligoastrocytoma $(n=1)$ & Diffuse astrocytoma IDH'mut & 1 \\
\hline Total & & 124 \\
\hline
\end{tabular}

WHO, World Health Organization; IDH'm , isocitrate dehydrogenase mutant; IDH' ${ }^{\text {wt }}$ NOS, isocitrate dehydrogenase wildtype, not otherwise specified.

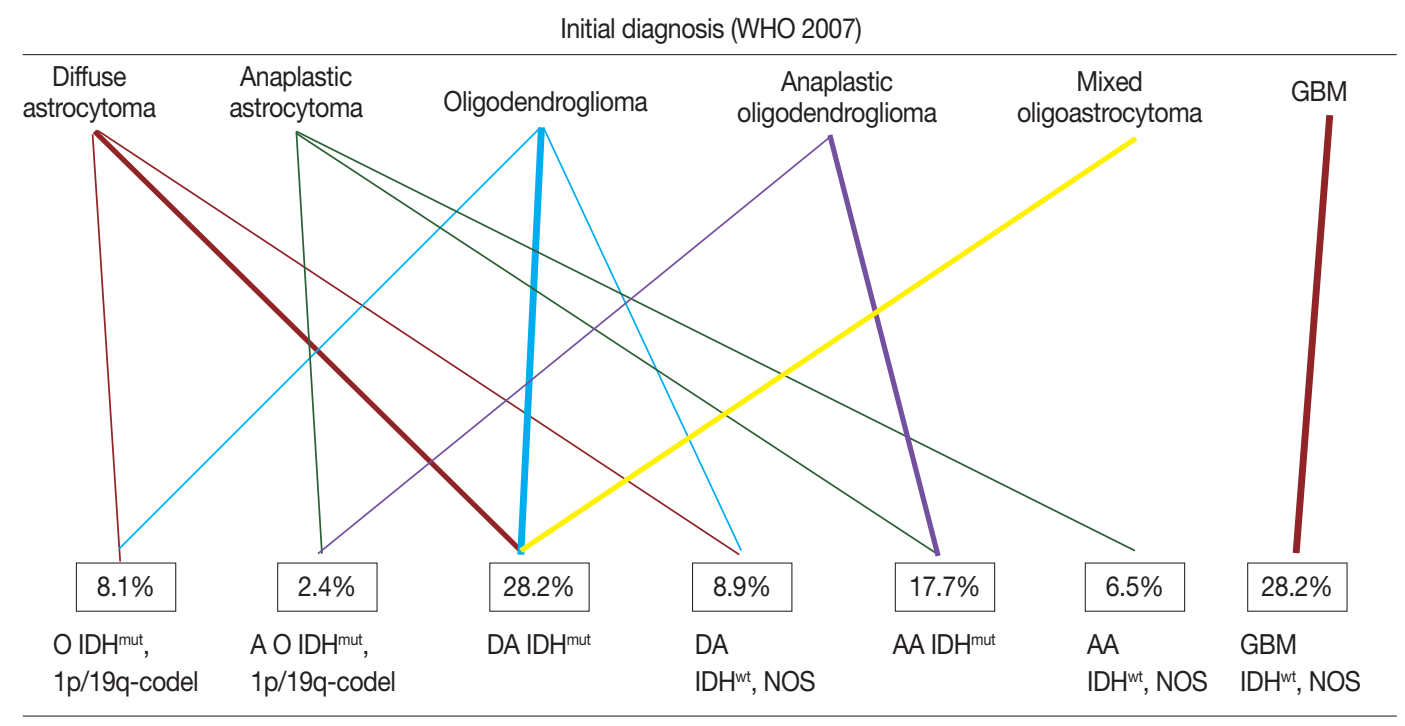

Integrated diagnosis (WHO 2016)

Fig. 3. Change in diagnosis after applying molecular genetics integrated diagnostic criteria according to the updated $2016 \mathrm{WHO}$ classification. WHO, World Health Organization; IDH ${ }^{\text {mut }}$, isocitrate dehydrogenase mutant; IDH ${ }^{\text {wt }}$, NOS, isocitrate dehydrogenase wildtype, not otherwise specified; 1p/19q codel, 1p/19q co-deleted.

omas $\mathrm{IDH}^{\text {mut }}$ and $1 \mathrm{p} / 19 \mathrm{q}$ co-deleted. One case of mixed oligoastrocytoma was reclassified as diffuse astrocytoma $\mathrm{IDH}^{\mathrm{mut}}$. The summary of integrated diagnosis is shown in Table 2 and Fig. 3.

In this study, 124 patients were reclassified as diffuse astrocytoma $\mathrm{IDH}^{\mathrm{mut}}, 28.2 \%(\mathrm{n}=35)$; diffuse astrocytoma $\mathrm{IDH}^{\mathrm{wt}}$, NOS, 8.9\% ( $\mathrm{n}=11)$; anaplastic astrocytoma $\mathrm{IDH}^{\text {mut }}, 17.7 \%(\mathrm{n}=22)$; anaplastic astrocytoma IDH ${ }^{\mathrm{wt}}$, NOS, $6.5 \%(\mathrm{n}=8)$; oligodendroglioma $\mathrm{IDH}^{\mathrm{mut}}$ and $1 \mathrm{p} / 19 \mathrm{q}$ co-deleted, $8.1 \%(\mathrm{n}=10)$; anaplastic oligodendroglioma $\mathrm{IDH}^{\mathrm{mut}}$ and $1 \mathrm{p} / 19 \mathrm{q}$ co-deleted, $2.4 \%(\mathrm{n}=3)$; and glioblastoma IDH ${ }^{\mathrm{wt}}$, NOS, $28.2 \%(\mathrm{n}=35)$. None of the patients developed glioblastoma IDH $^{\text {mut }}$ (Table 3).
There was a significant change in frequency of both oligodendroglial (34.7\% to $10.5 \%)$ and astrocytic $(37.1 \%$ to $60.7 \%)$ entities after reclassification according to the new 2016 WHO classification. The frequencies of 1p/19q co-deletion and IDH1 mutation were significantly higher in patients with low-grade tumors (grade II) than in patients with high-grade tumors (grades III and IV) (17.9\% vs $4.4 \%, \mathrm{p}<.05$ for $1 \mathrm{p} / 19 \mathrm{q}$ co-deletion and $80.4 \%$ vs $36.8 \%, \mathrm{p}<.001$ for IDH1 mutation). Notably, neither 1p/19q co-deletion nor IDH1 mutation was observed in patients with grade IV glioblastoma (Table 3). 
Table 3. Comparison between 2007 and 2016 WHO classification of diffuse gliomas

\begin{tabular}{|c|c|c|}
\hline $\begin{array}{l}\text { Original histological diagnosis according } \\
\text { to WHO } 2007\end{array}$ & $\begin{array}{l}\text { WHO } \\
\text { grade }\end{array}$ & No. (\%) \\
\hline \multicolumn{3}{|l|}{ Astrocytic tumors } \\
\hline Diffuse astrocytoma & $\|$ & $29(23.4)$ \\
\hline Anaplastic astrocytoma & III & $17(13.7)$ \\
\hline Glioblastoma & IV & $35(28.2)$ \\
\hline \multicolumn{3}{|l|}{ Oligodendroglial tumors } \\
\hline Oligodendroglioma & $\|$ & $26(21.0)$ \\
\hline Anaplastic oligodendroglioma & III & $16(12.9)$ \\
\hline Mixed oligoastrocytoma & $\|$ & $1(0.8)$ \\
\hline \multicolumn{3}{|l|}{ Integrated diagnosis according to updated WHO 2016} \\
\hline Diffuse astrocytoma IDH mut & $\|$ & $35(28.2)$ \\
\hline Diffuse astrocytoma IDH'wt, NOS & $\|$ & $11(8.9)$ \\
\hline Anaplastic astrocytoma IDHmut & III & $22(17.7)$ \\
\hline Anaplastic astrocytoma IDH'w , NOS & III & $8(6.5)$ \\
\hline Oligodendroglioma IDHmut, $1 p / 19 q$ co-deleted & $\|$ & $10(8.1)$ \\
\hline $\begin{array}{l}\text { Anaplastic oligodendroglioma IDHmut, 1p/19q } \\
\text { co-deleted }\end{array}$ & III & $3(2.4)$ \\
\hline Glioblastoma IDH'wt, NOS & IV & $35(28.2)$ \\
\hline
\end{tabular}

WHO, World Health Organization; IDH ${ }^{\text {mut }}$, isocitrate dehydrogenase mutant; $\mathrm{IDH} \mathrm{Ht}^{\mathrm{wt}}$, NOS, isocitrate dehydrogenase wildtype, not otherwise specified.

\section{Prognostic value of 2016 WHO classifications}

During follow-up, all-cause mortality occurred in $61.3 \%$ ( $\mathrm{n}=$ 76) of patients, and $38.7 \%(\mathrm{n}=48)$ of patients survived. Median OS was 13 months (95\% confidence interval, 10.2 to 15.7). The discriminative value of the 2007 and 2016 WHO classifications was tested using Cox proportional hazard regression model for all-cause mortality and summarized in Table 4. Oligodendroglioma and oligodendroglioma $\mathrm{IDH}^{\text {mut }}$ and 1p/19q co-deleted were selected as the reference diagnoses for 2007 and 2016 WHO classifications, respectively. The prognostic significance of the 2007 WHO classifications was compared with that of the new 2016 WHO classifications using Kaplan-Meier estimation. Both the 2007 and 2016 WHO classifications had significantly discriminative information for all-cause mortality $(\mathrm{p}<.001)$ (Fig. 4A, B). However, the new 2016 WHO classification had higher hazard ratio than the 2007 WHO classification (Table 4).

The new 2016 WHO classification showed a statistically significant survival advantage for grade II tumors compared with the 2007 WHO classification (Fig. 4C, D). Based on the 2007 WHO classification, no survival difference was found between patients with grade II tumors including those with oligodendroglioma, diffuse astrocytoma, and oligoastrocytoma $(\mathrm{p}=.437)$. However, the new 2016 WHO classification showed that oligodendroglioma $\mathrm{IDH}^{\text {mut }}$ and 1p/19q co-deleted and diffuse astrocytoma $\mathrm{IDH}^{\text {mut }}$ had better survival compared with diffuse astrocytoma IDH ${ }^{\mathrm{wt}}$, NOS in grade II tumors $(\mathrm{p}<.01)$. Both
Table 4. Discriminative value of 2007 and 2016 WHO classification based on Cox proportional hazard regression for all-cause mortality

\begin{tabular}{|c|c|c|c|}
\hline & $\mathrm{HR}$ & $95 \% \mathrm{Cl}$ & $p$-value \\
\hline \multicolumn{4}{|l|}{2007 WHO classification } \\
\hline Oligodendroglioma & $\mathrm{RF}$ & & \\
\hline Diffuse astrocytoma & 0.60 & $0.27-1.37$ & .227 \\
\hline Mixed oligoastrocytoma & 1.30 & $0.17-9.96$ & .802 \\
\hline Anaplastic astrocytoma & 2.16 & $1.01-4.65$ & .048 \\
\hline Anaplastic oligodendroglioma & 2.42 & $1.12-5.22$ & .025 \\
\hline Glioblastoma & 2.67 & $1.36-5.24$ & .004 \\
\hline \multicolumn{4}{|l|}{2016 WHO classification } \\
\hline $\begin{array}{l}\text { Oligodendroglioma IDHmut, } \\
1 p / 19 q \text { co-deleted }\end{array}$ & $\mathrm{RF}$ & & \\
\hline Diffuse astrocytoma IDH mut & 1.07 & $0.31-3.72$ & .913 \\
\hline $\begin{array}{l}\text { Anaplastic oligodendroglioma IDH }{ }^{\text {mut }} \text {, } \\
1 p / 19 q \text { co-deleted }\end{array}$ & 2.94 & $0.49-17.6$ & .239 \\
\hline Anaplastic astrocytoma IDH ${ }^{\text {wt }}$, NOS & 3.70 & $0.87-15.6$ & .075 \\
\hline Diffuse astrocytoma IDH ${ }^{w t}$, NOS & 4.13 & $1.02-16.8$ & .047 \\
\hline Anaplastic astrocytoma IDH'mut & 3.95 & $1.16-13.4$ & .028 \\
\hline Glioblastoma IDH'wt, NOS & 4.50 & $1.34-15.0$ & .015 \\
\hline
\end{tabular}

WHO, World Health Organization; HR, hazard ratio; Cl, confidence interval; $\mathrm{IDH} \mathrm{Hut}^{\text {, }}$, isocitrate dehydrogenase mutant; IDH'wt NOS, isocitrate dehydrogenase wildtype, not otherwise specified.

2007 and 2016 WHO classifications did not show any survival difference in patients with grade III and grade IV tumors $(\mathrm{p}=$ .777 and $\mathrm{p}=.936$, respectively) (Fig. 4E, F).

\section{DISCUSSION}

Our study results were summarized as follows: (1) the new 2016 WHO classification led to substantial changes in the diagnosis of diffuse gliomas, and (2) the new integrated histomolecular classification, based on molecular data, provided more valuable prognostic information.

Studies over the last two decades clearly demonstrated that the genetic basis of oncogenesis is important for the development of brain tumor entities and clarified their role in patients' prognosis. Boulay et al. ${ }^{14}$ and Hata et al. ${ }^{15}$ reported chromosomal changes in human gliomas. Notably, Mizoguchi et al. ${ }^{16}$ investigated the role of $1 \mathrm{p} / 19 \mathrm{q}$ co-deletion in patients with glioblastoma and its prognostic relation. Furthermore, several studies revealed the frequency of IDH mutation and its prognostic value in human gliomas. ${ }^{17-20}$ Based on these results, new integrated WHO CNS tumor classification was introduced to clinical practice in 2016; ; conceptual and practical advances were made over previous version. ${ }^{3}$ First, 2016 WHO classification is based on not only histological features but also genetic alterations of gliomas, such as $1 \mathrm{p} / 19 \mathrm{q}$ co-deletion and IDH mutation, for diffuse brain glioma classification. Second, molecular pathologic tests are essen- 
tial for the diagnosis of diffuse gliomas. The 2016 WHO classification of CNS tumors requires at least IDH1 immunohistochemistry and 1p/19q co-deletion status for the diagnosis of diffuse gliomas in addition to histologic evaluation. With regard
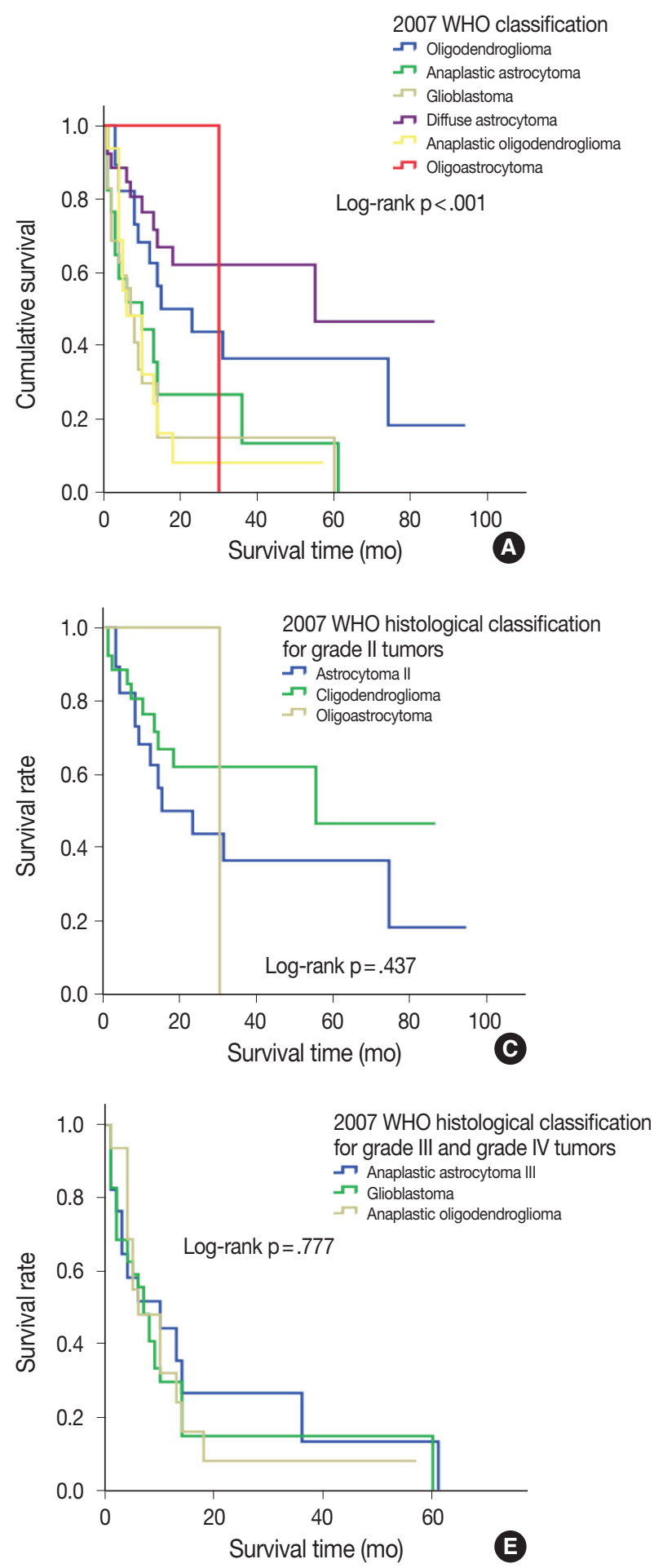

to IDH mutation, mutation analyses of both IDH-1 and IDH2 are recommended more than immunohistochemistry for detection of IDH1 hotspot mutation (p.R132H).

Mellai et al. ${ }^{21}$ reported that $\mathrm{IDH1}$ mutations consisted $98.5 \%$
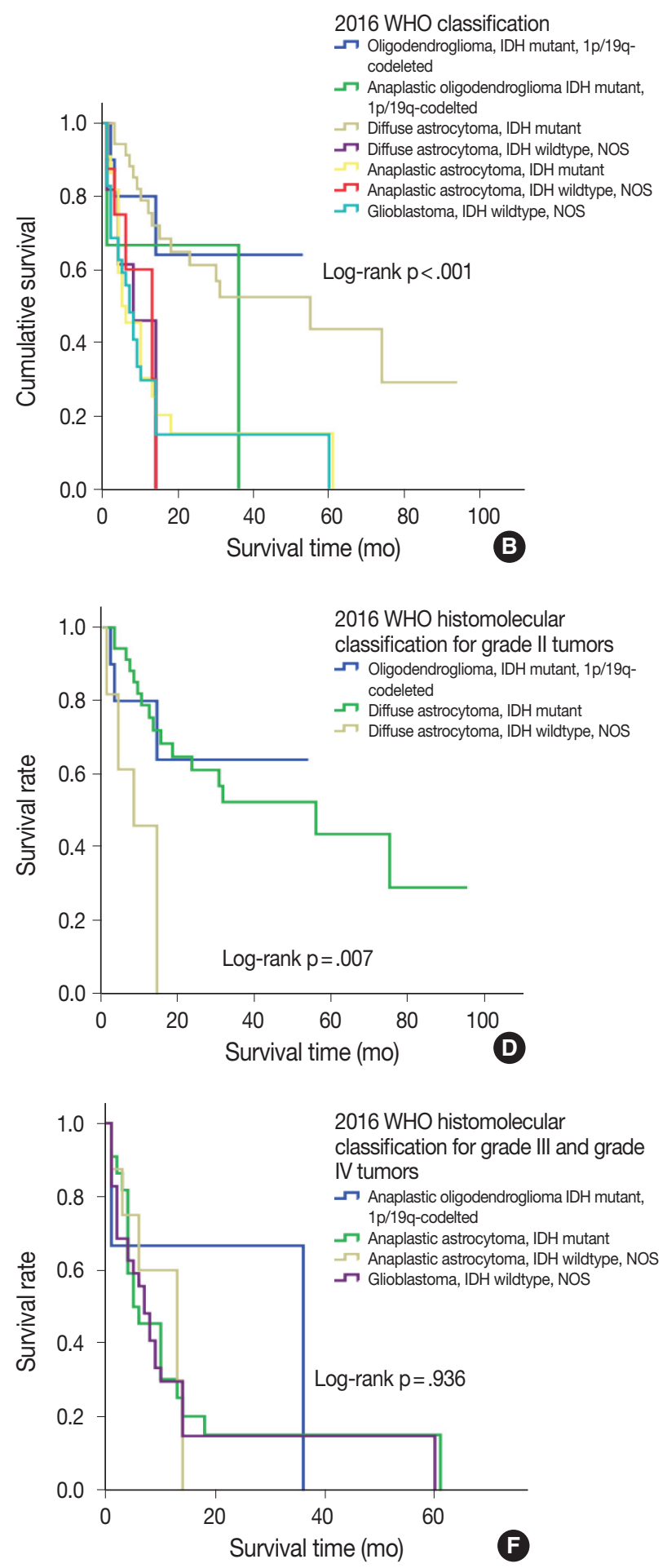

Fig. 4. Kaplan-Meier curves for overall survival according to the 2007 WHO classification (A, C, E) and 2016 WHO classification (B, D, F), respectively. (A, B) For all tumors. (C, D) For grade II tumors. (E, F) For grades III and IV tumors. WHO, World Health Organization. 
of all IDH mutations in gliomas. Among IDH1 mutations, 93.7\% were c.395G > A (p.R132H) which can be detected by $\mathrm{mIDH} 1^{\mathrm{R} 132 \mathrm{H}}$ antibody immunostaining. There was a statistically significant correlation between $\mathrm{mIDH} 1^{\mathrm{R} 132 \mathrm{H}}$ antibody immunostaining and the relevant mutation c.395G > A (p.R132H) (p $=.0001) \cdot{ }^{21}$ Different types of IDH1 gene mutations at codon R132 have been identified, of which c.395G > A (p.R132H) mutation accounts for about 93\%, c.394C > T (p.R132C) for $4 \%$, c.394C > A (p.R132S) for $1.5 \%$ in the different types of gliomas. ${ }^{17,22}$

The 2016 WHO classification of CNS tumors is a global standard, and it has better prognostic significance than traditional histological classification. To perform the molecular diagnosis of gliomas according to the 2016 WHO classification of CNS tumors, full assessment of IDH mutation status and 1p/19q analyses are mandatory. Operating a well-equipped molecular laboratory is a challenging situation in the developing countries including Mongolia. Expansion of facilities for molecular pathology is required to avoid the diagnosis of diffuse glioma, NOS. Nowadays pathology laboratories in developing countries struggle to provide specialized molecular tests, and it requires increase of medical costs.

In this study, we could reclassify Mongolian diffuse gliomas according to the new 2016 WHO classification. Reclassification revealed substantial changes in diagnosis of both oligodendroglial and astrocytic entities. For example, histologically astrocytic entities, which had both IDH mutation and 1p/19q co-deletion, were reclassified into oligodendroglial entities (3 of 46 patients), and oligodendroglial entities, without $1 \mathrm{p} / 19 \mathrm{q}$ co-deletion, were reclassified into astrocytic entities (30 of 42 patients). Furthermore, molecular subgroups, such as $\mathrm{IDH}^{\mathrm{mut}}$ and $\mathrm{IDH}^{\mathrm{wt}}$, NOS, were added to the diagnosis of diffuse glioma based on the results of immunohistochemical staining of IDH1. Similar results were observed in the French POLA cohort study by Tabouret et al. ${ }^{5}$

The previous studies suggest that $1 \mathrm{p} / 19 \mathrm{q}$ co-deletion or IDH mutation is a relatively early event during the development of glioma. ${ }^{23,24}$ As a result, the frequency of $1 \mathrm{p} / 19 \mathrm{q}$ co-deletion or IDH mutation could be higher in low-grade gliomas. In our study, the frequency of $1 \mathrm{p} / 19 \mathrm{q}$ co-deletion and IDH1 mutation were significantly higher in patients with low-grade gliomas than in those with high-grade gliomas ( $17.9 \%$ vs $4.4 \%, \mathrm{p}<.05$ and $80.4 \%$ vs $36.8 \% \mathrm{p}<.001$, respectively).

Reclassification of diffuse gliomas not only categorizes molecular subgroups of diffuse gliomas but it also has important prognostic value. Yan et al..$^{25}$ revealed that tumors with IDH mutation are distinctive genetically and clinically, and had better outcomes than those with wildtype IDH gene. Akagi et al. ${ }^{10}$ and Taburet et al. ${ }^{5}$ demonstrated that the new 2016 WHO classification has better prognostic value in terms of OS. In particular, $\mathrm{IDH}^{\text {mut }}$ is a strong prognostic marker and is associated with better survival compared with $\mathrm{IDH}^{\mathrm{wt}} 5,10$ However, the prognostic advantage of IDH1 $1^{\text {mut }}$ was only evident for low-grade gliomas in this study.

In our study, the 2016 WHO classification showed higher hazard ratio for OS than the 2007 WHO classification and reinforced the prognostic value of integrated histomolecular classification. The 2007 WHO classification did not show survival difference in grade II tumors, whereas 2016 WHO classification showed that oligodendroglioma, IDH ${ }^{\text {mut }}$ and 1p/19q co-deleted and diffuse astrocytoma $\mathrm{IDH}^{\mathrm{mut}}$ had better survival compared with diffuse astrocytoma IDH ${ }^{\mathrm{wt}}$, NOS in grade II tumors.

Finally, our study has several limitations. First, the total study population was relatively small compared with those in other similar studies. Therefore, further studies with a large sample size are required to confirm these findings. Second, there were some technical difficulties associated with FISH testing. In our study, 32 patients who were diagnosed before 2012 had no signal on FISH test. It could be caused by laboratory suboptimal conditions such as poor fixation of tissue and storage duration of tissue blocks. Therefore, we reclassified those cases based on histological pattern and IDH1 mutation status only without 1p/19q co-deletion information. Third, IDH mutation status has been investigated only by immunohistochemistry, and no further molecular analysis for IDH mutation was performed in cases of diffuse gliomas that are immunohistochemically negative for IDH1 R132H mutation. Therefore, we could not avoid the diagnosis of diffuse gliomas, IDH-wildtype, NOS.

This is the first study to report the reclassification of Mongolian diffuse gliomas according to the revised 2016 WHO CNS tumor classification. There has been no study regarding pathological classification of brain gliomas according to the 2007 WHO classification as well as survival analysis of gliomas in Mongolia. In spite of several technical limitations, our results were still similar to those of previous publications. Additionally, we have confirmed that the revised 2016 WHO CNS tumor classification is also feasible for Mongolian diffuse gliomas and that it has prognostic significance in Mongolian patients with diffuse gliomas.

\section{ORCID}

Enkhee Ochirjav: https://orcid.org/0000-0003-4525-2378

Bayarmaa Enkhbat: https://orcid.org/0000-0003-3163-4316 
Tuul Baldandorj: https://orcid.org/0000-0001-5932-1114

Gheeyoung Choe: https://orcid.org/0000-0001-6547-5603

\author{
Author Contributions \\ Conceptualization: EO, GC. \\ Data curation: EO. \\ Formal analysis: EO. \\ Investigation: $\mathrm{EO}$. \\ Methodology: EO, GC. \\ Project administration: $\mathrm{BE}$. \\ Resources: EO, TB, BE. \\ Supervision: GC. \\ Validation: EO, TB. \\ Visualization: EO. \\ Writing—original draft: EO, TB. \\ Writing — review \& editing: BE, GC.
}

\section{Conflicts of Interest}

The authors declare that they have no potential conflicts of interest.

\section{Acknowledgments}

This study was conducted in the National Center for Pathology; Department of Pathology, MNUMS; Department of Neurosurgery, State Third Central Hospital; and Department of Pathology, Seoul National University Bundang Hospital. We gratefully acknowledge their support.

\section{REFERENCES}

1. Cancer Genome Atlas Research Network, Brat DJ, Verhaak RG, et al. Comprehensive, integrative genomic analysis of diffuse lowergrade gliomas. N Engl J Med 2015; 372: 2481-98.

2. Reuss DE, Kratz A, Sahm F, et al. Adult IDH wild type astrocytomas biologically and clinically resolve into other tumor entities. Acta Neuropathol 2015; 130: 407-17.

3. Louis DN, Ohgaki H, Wiestler OD, et al. The 2007 WHO classification of tumours of the central nervous system. Acta Neuropathol 2007; 114: 97-109.

4. Hirose Y, Sasaki H, Abe M, et al. Subgrouping of gliomas on the basis of genetic profiles. Brain Tumor Pathol 2013; 30: 203-8.

5. Tabouret E, Nguyen AT, Dehais C, et al. Prognostic impact of the 2016 WHO classification of diffuse gliomas in the French POLA cohort. Acta Neuropathol 2016; 132: 625-34.

6. Hainfellner J, Louis DN, Perry A, Wesseling P. Letter in response to David N. Louis et al, International Society of Neuropathology-
Haarlem Consensus Guidelines for Nervous System Tumor Classification and Grading, Brain Pathology, doi: 10.1111/bpa.12171. Brain Pathol 2014; 24: 671-2.

7. Louis DN, Perry A, Reifenberger G, et al. The 2016 World Health Organization classification of tumors of the central nervous system: a summary. Acta Neuropathol 2016; 131: 803-20.

8. Gupta A, Dwivedi T. A simplified overview of World Health Organization classification update of central nervous system tumors 2016. J Neurosci Rural Pract 2017; 8: 629-41.

9. Wesseling P, Capper D. WHO 2016 classification of gliomas. Neuropathol Appl Neurobiol 2018; 44: 139-50.

10. Akagi Y, Yoshimoto K, Hata N, et al. Reclassification of 400 consecutive glioma cases based on the revised 2016WHO classification. Brain Tumor Pathol 2018; 35: 81-9.

11. Iuchi T, Sugiyama T, Ohira M, et al. Clinical significance of the 2016 WHO classification in Japanese patients with gliomas. Brain Tumor Pathol 2018; 35: 71-80.

12. Pisapia DJ. The updated World Health Organization glioma classification: cellular and molecular origins of adult infiltrating gliomas. Arch Pathol Lab Med 2017; 141: 1633-45.

13. Louis DN, Ellison DW, Brat DJ, et al. cIMPACT-NOW: a practical summary of diagnostic points from Round 1 updates. Brain Pathol 2019; 29: 469-72.

14. Boulay JL, Stiefel U, Taylor E, Dolder B, Merlo A, Hirth F. Loss of heterozygosity of TRIM3 in malignant gliomas. BMC Cancer 2009; 9: 71.

15. Hata N, Yoshimoto K, Yokoyama N, et al. Allelic losses of chromosome 10 in glioma tissues detected by quantitative single-strand conformation polymorphism analysis. Clin Chem 2006; 52: 370-8.

16. Mizoguchi M, Yoshimoto K, Ma X, et al. Molecular characteristics of glioblastoma with 1p/19q co-deletion. Brain Tumor Pathol 2012; 29: 148-53.

17. Balss J, Meyer J, Mueller W, Korshunov A, Hartmann C, von Deimling A. Analysis of the IDH1 codon 132 mutation in brain tumors. Acta Neuropathol 2008; 116: 597-602.

18. Hartmann C, Meyer J, Balss J, et al. Type and frequency of IDH1 and $\mathrm{IDH} 2$ mutations are related to astrocytic and oligodendroglial differentiation and age: a study of 1,010 diffuse gliomas. Acta Neuropathol 2009; 118: 469-74.

19. Ichimura K, Pearson DM, Kocialkowski S, et al. IDH1 mutations are present in the majority of common adult gliomas but rare in primary glioblastomas. Neuro Oncol 2009; 11: 341-7.

20. Sanson M, Marie Y, Paris S, et al. Isocitrate dehydrogenase 1 codon 132 mutation is an important prognostic biomarker in gliomas. J Clin Oncol 2009; 27: 4150-4.

21. Mellai M, Piazzi A, Caldera V, et al. IDH1 and IDH2 mutations, im- 
munohistochemistry and associations in a series of brain tumors. J Neurooncol 2011; 105: 345-57.

22. von Deimling A, Korshunov A, Hartmann C. The next generation of glioma biomarkers: MGMT methylation, BRAF fusions and IDH1 mutations. Brain Pathol 2011; 21: 74-87.

23. Bigner SH, Matthews MR, Rasheed BK, et al. Molecular genetic as- pects of oligodendrogliomas including analysis by comparative genomic hybridization. Am J Pathol 1999; 155: 375-86.

24. Cohen AL, Holmen SL, Colman H. IDH1 and IDH2 mutations in gliomas. Curr Neurol Neurosci Rep 2013; 13: 345.

25. Yan H, Parsons DW, Jin G, et al. IDH1 and IDH2 mutations in gliomas. N Engl J Med 2009; 360: 765-73. 